\title{
De Novo and recurrent thrombotic microangiopathy (TMA) after renal transplantation: current concepts in management
}

\begin{abstract}
Thrombotic microangiopathy (TMA) is a well-recognized complication of kidney transplantation that leads frequently to allograft failure. This serious outcome depends greatly on the underlying etiology as well as the timing of therapeutic interventions. TMA syndromes may occur with no previous history of TMA, i.e., de novo TMA, mostly due to medications or infection, or more frequently recurs after kidney transplantation i.e., recurrent TMA in patients with ESRF due to the atypical hemolytic uremic syndrome (aHUS). On the other hand, patients with shiga-toxin induced HUS (classic HUS), particularly in childhood has a favorable prognosis. One of the fundamental tools of management of this disease is the genetic screening for abnormal mutations, determination of which will recognize the tools of therapy and consequently outcome of the disease to a large extent. While patients with $\mathrm{CFH}$ and CFI mutations have a worse prognosis, other patients with MCP mutations-for example- have a more favorable prognosis. Accordingly, plan of therapy can be thoroughly drawn with a better chance of cure. Unfortunately, the successful use of the biological agent "eculizumab", an anti-C5 agent, in some of these syndromes is largely impeded by its high cost linked to its use as a life-long therapy. However, a new therapeutic option has been recently admitted ameliorating this drawback and improve the cost-effectiveness balance.
\end{abstract}

Volume 9 Issue I - 202|

\author{
Fedaei Abbas, ${ }^{1,2}$ Sara Fedaei Abbas ${ }^{3}$ \\ 'Jaber El Ahmed Military Hospital, Nephrology department, \\ Kuwait \\ ${ }^{2}$ Faculty of Health and Science, University of Liverpool, Institute \\ of Learning and Teaching, School of Medicine, Liverpool - United \\ Kingdom \\ ${ }^{3}$ College of Medicine, Ain Shams University, Egypt

\begin{abstract}
Correspondence: Fedaei Abbas, Consultant Nephrologist, Jaber El Ahmed Military Hospital,Nephrology department, P.O. Box 454, Safat I3005, Kuwait, Tel+965 9752909|,

Email Fedaeyabas2009@gmail.com,

Fedaeybbas2008@yahoo.com
\end{abstract}

Received: December 29, 2020 | Published: February 26, 202

Keywords: thrombotic microangiopathy- de novo tma - recurrent tma - renal allograft

Abbreviations: TMA, thrombotic microangiopathy; MAHA, microangiopathic hemolytic anemia; HUS, hemolytic uremic syndrome; aHUS, atypical hemolytic uremic syndrome; TTP, thrombotic thrombocytopenic purpura; KTR, kidney transplant recipients; $\mathrm{CFH}$, complement factor h; CFI, complement factor I; MCP, membrane cofactor protein; THBD, gene encoding thrombomodulin; PE, Plasmapheresis; mTORi, Mammalian target of rapamycin inhibitors

\section{Introduction}

The evolution of thrombotic microangiopathy (TMA) after renal transplantation, either de novo or recurrent disease, is a welldocumented complication that not only affects allograft function, but also adversely influences patient and allograft survival. ${ }^{1}$ Management of this devastating disease depends largely on the underlying etiology. While the shiga toxin-associated HUS (classic HUS) is usually selflimited with a favorable prognosis, the complement-mediated HUS, (atypical HUS, aHUS) usually carries worse outcome that requires a more sophisticated therapeutic approach. ${ }^{1}$ In 1981, Shulman et al. ${ }^{4}$ described an association between cyclosporine (CyA) and TMA for the first time. ${ }^{2}$ TMA was also linked to the most used CNI, tacrolimus with an estimated incidence of approximately 3-14 \% in some series ${ }^{3}$ and increases up to $25 \%$ in others. ${ }^{4}$ aHUS is a systemic disease involving the kidney and recurs in up to $100 \%$ of renal transplant, with poor allograft outcome. ${ }^{8}$ Pathogenesis is due to dysregulation of the alternative complement pathway that triggers uncontrolled cleavage of terminal complement protein C5 with excessive C5b-9 complex production. ${ }^{5}$ This cascade results in endothelial injury, increased expression of adhesion molecules that consequently followed by fibrinrich micro-thrombi with an end-organ ischemia, thrombocytopenia and microangiopathic hemolytic anemia (MAHA). ${ }^{5-7}$ In this review we will shed some light on the recent concepts in management of different forms of TMA.
Definition: Thrombotic microangiopathy (TMA) refers to a histopathologic entity that includes vessel wall thickening (arterioles and capillaries), intraluminal thrombi and vessel luminal occlusion. The resultant platelet and RBCs consumption at the level of microvasculature of vital organs including the kidney, leads to thrombocytopenia and microangiopathic hemolytic anemia (MAHA). Two clinical situations with overlapping features were recognized; thrombotic thrombocytopenic purpura (TTP) and hemolytic uremic syndrome (HUS) with neurological features are essential features of the TTP but not in the HUS. ${ }^{9}$

Etiology: KTR can develop HUS either de novo or as a recurrent disease:

I. De novo HUS: This includes causes of TMA in native kidneys and transplant specific causes as well. Medications such as CNI, ${ }^{10,11}$ viral infection, ischemia/reperfusion injury and Antibodymediated rejection (AMR) $)^{12-16}$ are all among transplantation related causes. De novo HUS secondary to genetic mutations in complement-regulatory proteins can develop less frequently. ${ }^{17}$

II. Recurrent HUS: This type of HUS is almost always due to dysregulation of complement secondary to genetic mutations in complement proteins that regulate the alternative pathway. They are usually called the "complement-mediated" HUS or the atypical HUS (aHUS). ${ }^{18}$

\section{Prevalence}

De novo HUS: the reported rate of de novo HUS is about 3-14\% of KTR. ${ }^{3,19,20}$ The following list includes factors that are particularly encountered in KTR: ${ }^{21-23}$
I. AMR.
II. Infections: e.g., CMV, HIV, parvovirus B19. 


\section{Medications: e.g., CNI, mTOR inhibitors and valacyclovir.}

- Switching the patients on CyA immunosuppression to tacrolimus can reverse the CyA-induced HUS in more than $80 \%$ of patients. ${ }^{11}$ Data is limited as regard the evidence clarifying this response.

Recurrent HUS: While more than $90 \%$ of the childhood-onset HUS is mostly related to the Shiga toxin-producing E. coli with a recurrence rate of less than $1 \%$ of patients after renal transplantation, ${ }^{24}$ many patients with complement-mediated HUS (aHUS) have recurrence after transplantation. The reported rate of recurrence in patients on dialysis secondary to HUS ranges between 25 and $50 \%{ }^{25-}$ ${ }^{28}$ However, recurrence is uncommon in infection-related HUS. On the other hand, the estimated rate of recurrence for complementmediated HUS is very frequent and depends to a great extent on the type of genetic mutations. ${ }^{24}$ While the estimated rate of recurrence has been reported to be $50-100 \%$ in mutations involved CFH and CFI, the reported rate is only $15-20 \%$ in mutation involving membrane cofactor protein $(\mathrm{MCP}) .{ }^{24} \mathrm{~A}$ given explanation for this low recurrence incidence of MCP-associated HUS is that MCP is highly expressed in the kidney, so that it is rapidly restored after transplantation of a new graft. ${ }^{29}$

Other reported risk factors of HUS recurrence include:

\section{CNI use. \\ II. Living related donor kidney.}

III. Short duration between HUS onset and the start of dialysis. ${ }^{30}$

Clinical presentation: Both de novo and recurrent HUS have a similar presentation (microangiopathic hemolytic anemia (MAHA), thrombocytopenia and AKI). Low platelet count, increased serum creatinine with evidence of hemolysis (reticulocytosis and schistocytes on peripheral smear and increased LDH) are usually encountered. A hematuria/proteinuria syndrome can be also seen. Nevertheless, this presentation is not universal, as some patients may present with only graft dysfunction with abnormal urine analysis., ${ }^{3,30}$ As regard timing of presentation, de novo HUS typically presents within the first three months post-transplant, ${ }^{24}$ while the recurrent HUS usually presents within days to weeks. ${ }^{31,32}$

Diagnosis: Any KTR presented with allograft dysfunction, should raise the possibility of HUS diagnosis, particularly so, if there is associated hemolytic anemia with drop of the platelet count. However, final diagnosis is ultimately documented by tissue diagnosis.

Pathology: the typical histopathological finding includes glomerular and arteriolar thrombosis, intra-capillary engorgement with RBCs and RBCs fragments, basement membrane detachment, endothelial swelling with glomerular ischemia. With healing of the arteriolar walls, an onion-skin hypertrophy supervenes Figure $(1,2){ }^{6}$

Two pathological entities in tissue biopsy can be encountered in allograft biopsy:

I. Cyclosporine nephrotoxicity: proximal tubular vacuolations with obliterative arteriopathy.

II. Acute AMR: intraluminal thrombi, circulating DSA and C4d staining of peritubular capillary.

Differential diagnosis: of AMR from HUS is difficult, as both conditions share in allograft dysfunction and resistance to antirejection therapy. However, the presence of predominant endarteritis with global involvement of the whole vascular tree of the graft is characteristic of the acute AMR. ${ }^{6}$

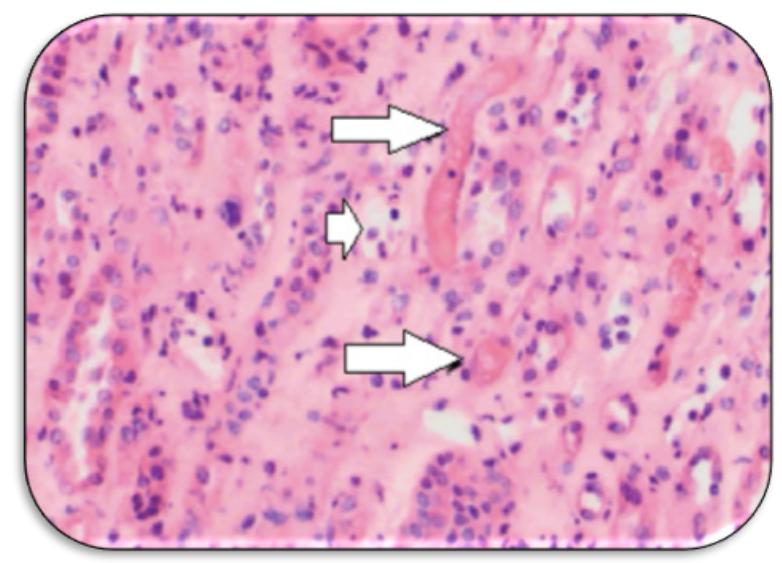

Figure I Medullary region of allograft biopsy obtained at onset of HUS symptoms with small vascular thrombi (arrows) and scattered dilated capillaries with peritubular capillaritis (short arrow) (Adapted from: Wrenn SM et al. ${ }^{36}$ open access).

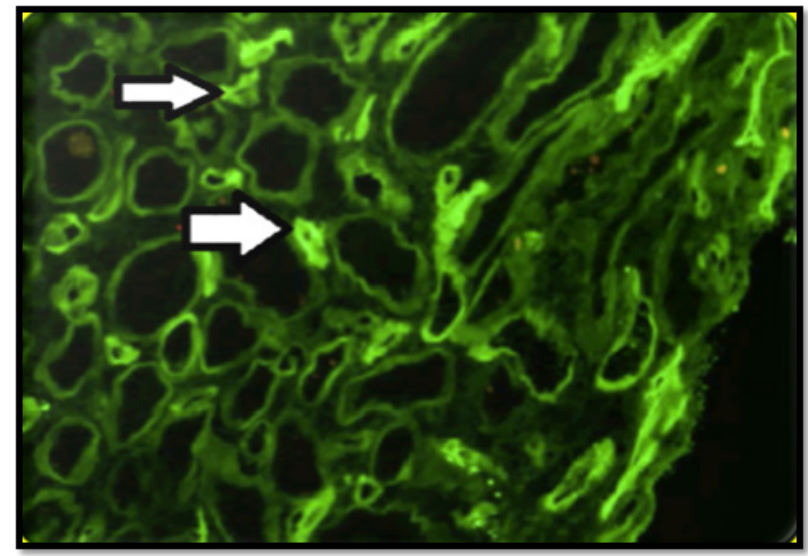

Figure 2 C4d IF staining of less than $50 \%$ of the peritubular capillaries (arrows), noted on the initial allograft biopsy. (Adapted from: Wrenn SM et al. ${ }^{36}$ open access).

Location: In about $30 \%$ of cases, TMA is strictly confined to allograft tissues with no associated manifestations of hemolysis or platelet count drop. In this situation, tissue diagnosis will be the only resort. Any young KTR presented with severe hypertension associated with decline in allograft function, should raise the possibility of TMA. ${ }^{3}$

Pathogenesis: An endothelial cell injury associated with imbalance between thrombotic and antithrombotic factors at the level of microvasculature has been postulated to be the culprit mechanism. Risk of TMA appears to be highest at the first three months posttransplant, with females and elderly patients appear to be more vulnerable. ${ }^{4}$

Classic HUS: Internalization of the Shiga toxin occurs in the endothelial cells, which is followed by activation and through cytokine release and endothelial damage, could develop a cascade of endothelial disruption, platelet clustering and thrombosis. The latter is the hallmark of TMA. ${ }^{33}$

CNI related: through potent vasoconstriction, endothelial toxicity, prothrombotic and antifibrinolytic criteria, ${ }^{28,34} \mathrm{CNI}$ can precipitate HUS. CyA and tacrolimus have the ability to up-regulate the production of vasoconstrictors elements e.g., endothelin-1 and angiotensin II that results in evolution of a pro-coagulation state that trigger platelet aggregation and thrombosis. ${ }^{28}$ 
Two different types of TMA have been described by Schwimmer and his associates (2003) in their retrospective study: systemic (62\%) and a localized type (38\%) without systemic extension. ${ }^{3}$ Graft loss have been more observed with the systemic form (more aggressive and disseminated disease), as compared with the localized type. ${ }^{3}$

AMR and HUS: It was Satoskar et al. ${ }^{34}$ who first admitted AMR as a cause of TMA. They found $55 \%$ of their biopsies were C4d positive. Another study found $88 \%$ of biopsies had C4d deposits..$^{35}$ Finally, both $\mathrm{C} 4 \mathrm{~d}$ staining of peritubular capillaries and DSA express the twofundamental links between TMA and AMR. ${ }^{6,36}$

Genetic predisposition: The absence of clear evidence of any HUS manifestation before transplant denotes de novo HUS diagnosis, however, Broeders et al. ${ }^{37}$ describes a case of silent polymorphism of factor " $\mathrm{H}$ " that present as de novo HUS after transplant. ${ }^{37}$ So, genetic predisposition to aHUS may be hidden in a "silent status" until clinically unmasked through an inciting factor (second hit) like transplant surgery or an associated infection..$^{38}$

Risk factors of de novo TMA: the following factors have been considered:

I. CNI: a reported incidence of $4-15 \%$ with $43 \%$ graft survival, have been reported to develop de novo TMA with high doses of CyA therapy. ${ }^{9,39}$ About $1 \%$ of renal transplant recipient receiving FK506 can develop de novo TMA.For CNI-related TMA the following mechanisms have been postulated:

\section{Potent vasoconstriction}

II. Endothelial toxicity.

III. Prothrombotic and antifibrinolytic activity.

The mTORi: Rapamycin has reported to be associated with de novo TMA. ${ }^{40}$

I. Donors after cardiac death: has been associated with posttransplant TMA. ${ }^{6}$

II. Prolonged warm ischemia: the resultant endothelial injury in the graft may increase the antigenic presentation that can induce acute rejection and TMA. ${ }^{6}$

III. Infection: e.g., viral infection (e.g., CMV). ${ }^{6}$

IV. De novo carcinoma, AMR, scleroderma, and antiphospholipid syndrome. ${ }^{6}$

V. Genetic mutations: mutation in $\mathrm{CFH}, \mathrm{CFI}$ and $\mathrm{CFH} / \mathrm{CFI}$ combination have been associated in $29 \%$ of patients with de novo TMA. ${ }^{18}$

VI. DSA: may be included in HUS evolution through an alternative mechanism of platelet activation that results in HUS with decline in allograft function due to the associated AMR. Some authors postulated that both TMA/HUS are likely linked to AMR under umbrella of genetic as well as acquired risk factors, with an end result of appearance of this devastating variant of allograft rejection. ${ }^{36}$

VII. Factor V Leiden: is considered by some investigators as a culprit factor among constellation of genetic defects that share in TMA evolution, especially with CNI exposition. ${ }^{41}$

Role of complement abnormality in aHUS recurrence: the outcome of aHUS is largely dependent on the type of complement aberrations. ${ }^{34,42-44}$

\section{Worse outcome}

I. Kidney transplant in patients with CFH mutations is usually complicated by a high rate of recurrence $(76 \%)$ with a high incidence of graft loss $(86 \%)$ as compared with those with CFH free mutations. ${ }^{31}$

II. The rate of recurrence increased to $92 \%$ in KTR with CFI mutations. Graft loss is $85 \%$ of the recurrent cases.

III. Moreover, patients with $\mathrm{C} 3$ and $\mathrm{CFB}$ mutation are also associated with a high rate of recurrence. ${ }^{6}$

\section{Better outcome}

I. Patients with "anti-CFH autoantibodies" have better outcome. $^{6}$

II. Membrane cofactor protein (MCP) mutations: $\mathrm{MCP}$ is a membrane-associated regulator that control complement activity. Recurrence of aHUS is rare as allograft endothelial cells express NORMAL MCP. ${ }^{6}$

III. Thrombomodulin (THBD) mutation has low risk of recurrence, as thrombomodulin is a transmembrane protein that resembles MCP. ${ }^{39}$

\section{Combined mutations}

It is difficult to interpret due to rarity of cases. However, heterozygous mutations in CFI/MCP and CFH/CFI were devoid of recurrence after renal transplantation, while other combinations of $\mathrm{CFH} / \mathrm{CFI}$ mutations and another with three mutations in $\mathrm{MCP} / \mathrm{CFH} /$ CFI have been reported to recur after transplantation. ${ }^{44,45}$

\section{Prognosis}

Prognosis of de novo is usually better than that of recurrent HUS. ${ }^{4}$ HUS has also a favorable prognosis if the lesions were confined to the glomeruli (localized form). ${ }^{46}$ The one- and five-year's survival rates of KTR with ESRF due to HUS were reported to be lower with HUS recurrence as compared with those without recurrence in one study (33\% versus $57 \%$ at one year and $19 \%$ versus $57 \%$ at five years). ${ }^{32}$

De novo versus recurrent TMA: the fundamental differences between de novo and recurrent TMA are summarized in (Table 1).

Prevention of HUS recurrence: to prevent HUS recurrence the following therapeutic approaches should be adopted:

Donor selection: avoid living related donor kidney for a patient developed ESRD due to complement-mediated HUS, the following explanations have been suggested:

I. A documented high rate of recurrence. .4,47 $^{24}$

II. Nephrectomy can trigger HUS in susceptible subjects. ${ }^{42}$

III. Negative genetic testing does NOT guarantee the absence of mutations, as some patients may have more than one mutation. ${ }^{9}$

IV. Genetic screening for mutations: before transplant, all patients on dialysis due to HUS and candidate for renal transplantation should be screened for the genetic mutations, as a sole dependence on clinical features alone can be misleading. ${ }^{29}$

V. Prophylactic strategies: for complement-mediated HUS due to genetic mutations the following interventions have been suggested: 
VI. For KTR receiving living unrelated donor kidney: eculizumab $900 \mathrm{mg}$ is given $24 \mathrm{~h}$. preoperative and in day 7, 14, and 21 then $1200 \mathrm{mg}$ every two weeks thereafter. Booster doses (900$1200 \mathrm{mg}$ ) should be given in presence of HUS triggering factors e.g., surgery or infection. ${ }^{29}$

VII. For KTR receiving deceased donor kidney: start ATG induction followed by $900 \mathrm{mg}$ eculizumab at day three postoperative, then $900 \mathrm{mg}$ weekly for three weeks, then $1200 \mathrm{mg}$ every two weeks thereafter. ${ }^{29}$

VIII. Combined PE with immunosuppression with corticosteroids and/or "rituximab" have been successfully tried in anti-CFH autoantibodies to get antibody titer decline and prevent HUS recurrence. ${ }^{48,49}$

Table I Main differences between de novo and recurrent HUS

\begin{tabular}{|c|c|c|c|}
\hline No & Item & De novo HUS & Recurrent HUS (aHUS). \\
\hline ( 1$)$ & Etiology & $\begin{array}{l}\text { Medications, e.g., CNI, }{ }^{10,11} \text { viral infection, } \\
\text { ischemia /reperfusion injury \& AMR. }\end{array}$ & $\begin{array}{l}\text { Dysregulation of complement activation due to genetic defects in the } \\
\text { alternative pathway (complement-mediated) or atypical HUS. } .^{18}\end{array}$ \\
\hline (2) & Timing & $\begin{array}{l}\text { Typically, within the first } 3 \text { months post- } \\
\text { transplant. }{ }^{24}\end{array}$ & Present within days to weeks after transplant..$^{31,32}$ \\
\hline (3) & Prevalence & About $3-14 \%$ of KTR. ${ }^{3,19,20}$ & $\begin{array}{l}\text { Recurrence rate: } \\
\text { - In patients on dialysis due to HUS: } 25-50 \% .^{25-28} \\
\text { - 50- } 100 \% \text { in mutations involved CFH \& CFI. } \\
\text { - Only I5-20\% in mutations involving MCP. }{ }^{24}\end{array}$ \\
\hline (4) & Pathology: & $\begin{array}{l}\text { Glomeruloarteriolar thromb-osis, capillary } \\
\text { engorgement, endothelial swelling. }\end{array}$ & - Same. \\
\hline (5) & Clinical presentation & MAHA, thrombocytopenia and AKI. & Similar presentation. \\
\hline \multirow{3}{*}{ (6) } & & & 2] CFI mutations: recurrence $92 \%$, Graft loss: $85 \%$. \\
\hline & & & 3] C3 \& CFB mutations: high rate of recurrence. ${ }^{6}$ \\
\hline & & & $\begin{array}{l}\text { II. Better outcome: } \\
\text { I] Anti-CFH autoantibodies: better outcome. } \\
\text { 2] MCP mutations: recurrence of aHUS is rare. }{ }^{6} \\
\text { 3] THBD mutations: low risk of recurrence. }{ }^{39}\end{array}$ \\
\hline (7) & Prevention & Avoid "risk factors" (see below). & Eculizumab (see below for more details). \\
\hline (8) & Treatment & $\begin{array}{l}\text { Hold the culprit drug. } \\
\text { PE. } \\
\text { Eculizumab (if associated with genetic } \\
\text { abnormality). }\end{array}$ & $\begin{array}{l}\text { Eculizumab (see the detailed protocol). } \\
\text { Bortezomib. } \\
\text { Rituximab. } \\
\text { Eculizumab "upon recurrence". } 7\end{array}$ \\
\hline
\end{tabular}

\section{Therapy of thrombotic microangiopathy (TMA)}

\section{General concepts}

TMA is a clinical phenotype that encompasses thrombotic thrombocytopenic purpura (TTP), shiga toxin-associated HUS (classic HUS) and atypical HUS (aHUS). While TTP responds well to plasma exchange (PE) and shiga toxin-associated HUS can be managed by supportive measures, the atypical HUS (aHUS), on the other hand, is a life-threatening event that necessitates more aggressive therapeutic maneuvers. ${ }^{8}$ Atypical HUS (aHUS) is a devastating disease presents usually with thrombocytopenia, hemolysis and renal failure. Prognosis is ultimately poor due to unlimited complement activity leading to thrombotic microangiopathy (TMA). While $50 \%$ of untreated patients progress to ESRF, $10 \%$ of them can be lost in its acute phase..$^{50} \mathrm{KTx}$ is a robust therapeutic option. Unfortunately, the high rate of recurrence with graft loss is a real threat. ${ }^{51}$ Trial of PE therapy has a very limited response with ultimate return to dialysis. ${ }^{52}$ The newly administrated biological agent, eculizumab (a complement 5 blocker) of increasing popularity due to rapid resumption of allograft function..$^{53}$ Moreover, KTx can be successfully performed under an umbrella of eculizumab prophylaxis. ${ }^{54}$ Considering the high cost of this biological agent is of great concern, especially so, with applying the "lifelong prophylactic strategy", ${ }^{55,56}$ consequently, alternative options have been recently addressed (see below).

\section{Treatment of de novo HUS}

$\mathrm{CNI} / \mathrm{mTORi}$ withdrawal or dose reduction to a lower trough level, whatever the suspected etiology. Considering these agents as common causes of HUS, their withdrawal/reduction can induce resolution of $d e$ novo HUS. ${ }^{21,28}$ Switching to tacrolimus is another option for patients receiving CyA.

I. If the disease progressed despite withdrawal of $\mathrm{CNI} / \mathrm{mTORi}$, start plasma exchange (PE) (1.5 volume FFP every $48 \mathrm{~h}$.) 
The beneficial effects of PE may be attributed to the removal of platelet-aggregating factors like thromboxane A2 with simultaneous replenishing the missing factors. ${ }^{20}$

II. For patients refractory to PE, start eculizumab $900 \mathrm{mg}$ IV Weekly for 4 weeks, followed by $1200 \mathrm{mg}$ every two weeks.

III. Genetic screening for mutations (e.g., in complement factor $\mathrm{H}$ or I) associated with complement-mediated HUS, should be performed:

IV. Negative results for culprit mutations: hold eculizumab and monitor closely for other triggering factors e.g., CMV and E. coli infection.

V. Positive mutations screening: continue eculizumab indefinitely (see below for other options). ${ }^{29}$

VI. Vaccinations for life threatening infections e.g., Neisseria meningitis, S. pneumoniae and Haemophiles influenza type B (Hib), should be performed for patients on eculizumab therapy. ${ }^{29}$

VII. Further therapeutic options include e.g., belatacept, rituximab have been described. ${ }^{57-60}$

Alternative options: Eculizumab with belatacept combination: the use of eculizumab combined with belatacept has been successfully applied as an alternative to $\mathrm{CNI}$ and to reverse a case of de novo aHUS in a patient with a heterozygous deletion in CFHR3-CFHR1 gene. Dedhia et al. ${ }^{8}$ presented a case of de novo aHUS after kidney transplantation in a patient with a heterozygous complement factor H-related protein (CFHR)3-CFHR1 deletion with a successful response to eculizumab therapy, with utilizing belatacept, a CD80binding fusion protein, for maintenance immunosuppression as an alternative to the $\mathrm{CNI}$ agent, tacrolimus. ${ }^{8}$

\section{Treatment of recurrent aHUS}

The significant role of a complement-mediated process as a culprit mechanism of HUS recurrence is now universally accepted. However, all other precipitating factors should be excluded before considering eculizumab therapy:

I. Withdraw CNI and mTOR inhibitors or at least reduce the dose to a lower trough level and continue steroids and antimetabolites. ${ }^{29}$

II. Exclude CMV, BK virus, parvovirus, and HIV infection through a PCR examination.

III. For patients with diarrhea, E. coli serotype O104:H4 (responsible of the reported E. coli outbreak-associated HUS) should be excluded through stool examination. ${ }^{29}$

IV. All patients missed the genetic screening for mutations in complement-associated aHUS, should have their genetic testing. ${ }^{29}$

Depending on the current available data, ${ }^{53,61-64}$ eculizumab therapy has its clear beneficial impact on the recurrence of aHUS disease. Many patients can be withdrawn from dialysis, with elevation of the platelet count and normalization of the LDH level. Therefore, all patients with recurrent HUS should receive eculizumab therapy as followed:

I. Dose: $900 \mathrm{mg}$ i.v. given weekly for four weeks followed by $1200 \mathrm{mg}$ every two weeks.
II. Target trough level of eculizumab $>100 \mathrm{mcg} / \mathrm{ml}$ is recommended. ${ }^{65}$

III. Duration: indefinite in recurrent aHUS as no randomized controlled trials for other options (see below).

IV. Daily HB, platelet count and LDH measurement in a hospitalized patient is advised, then regularly in the OPD clinic.

V. Monitor the response of eculizumab therapy through measuring the total hemolytic complement (CH50) before each dose for the $1^{\text {st }}$ four doses, a reasonable response of complete suppression is usually expected at a level below $10 \%{ }^{65}$

VI. Resistant cases should receive plasma exchange or plasma infusion (1.5 volume) every 48 hours, a booster dose of eculizumab is advised prior each plasma infusion $(300 \mathrm{mg}$ ) or after each PE session (600 mg).

VII. Meningococcal vaccination prior to commencing eculizumab therapy is advised to guard against a life-threatening infection with at least two weeks of prophylactic broad-spectrum antibiotics thereafter. ${ }^{29}$

Alternative options: In view of the high cost of eculizumab therapy, a recent discussion of experts in the last "EDTA 2017" conference, described a new strategy for better "cost-effectiveness" rates. The alternative options include the following:

Bortezomib: Depletion of plasma cells with the proteasome inhibitor "bortezomib" has been proposed as a new therapeutic option for management of the recurrent aHUS.

Eculizumab "upon recurrence": Recently, Brand (2017) and his colleagues presented a successful renal transplantation of aHUS patients without the need for eculizumab prophylaxis. ${ }^{7}$ Along 2.6 years follow-up these transplants and with avoiding risk factors that can trigger aHUS recurrence (e.g., surgery and viral infection), they succeeded to get a decline in recurrence rate up to $10 \%$. Moreover, they successfully achieved a reasonable cost-effectiveness rate with "eculizumab upon recurrence" strategy that depend primarily on monitoring the risk of recurrence through observation of the trigger factors. They also suggest monitoring the early markers e.g. thrombomodulin and soluble C5b-9 in urine. ${ }^{66}$

Both "eculizumab induction" and "lifelong maintenance therapy" strategies could result in an "over treatment" burden, which will not only has a negative impact on the "cost-effectiveness" balance but also will exaggerate the untoward effects burden.No evidence is currently available documented that curative eculizumab is less effective than prophylactic strategy. Therefore, the policy of "therapy upon recurrence" is preferable for a better cost-effectiveness balance. Furthermore, the need of early markers of disease recurrence appears to be urgently warranted. Promising markers of recurrence include thrombomodulin and soluble C5b-9 in urine. ${ }^{7}$ These markers are not only used for predicting HUS recurrence, but also can help in evaluating eculizumab response as well as drawing the best therapeutic plan. Fulfillment of these parameters in large cohorts of randomized studies can help achieve the best cost-effective strategy. ${ }^{66}$

Brand (2017) and his associates ${ }^{66}$ used the decision analytical approach (Markov modelling) to evaluate five strategies: (1) Dialysis therapy only; (2) Kidney transplantation (KTx) without eculizumab;(3) Eculizumab upon recurrence: KTx plus 3 mo. of eculizumab therapy in case of HUS recurrence; (4) Eculizumab 
induction: KTx plus 12 months of Eculizumab prophylaxis, and re-treatment with HUS recurrence, and (5) Eculizumab lifelong: KTx plus lifelong Eculizumab prophylaxis. They demonstrate that eculizumab therapy results in substantial benefit in QALYs (qualityadjusted life years). Moreover, applying the new suggested strategy of "eculizumab upon recurrence" is more efficacious in the concept of QALYs gain, as compared to both "eculizumab induction" and "lifelong eculizumab" prophylaxis. The latter two, however, can result in a higher cost-effective balance. Finally, living donor kidney transplantation for aHUS patients appears more feasible with no need for eculizumab prophylaxis as long as monitoring of trigger factors of recurrence was of utmost priority. ${ }^{66}$

\section{Conclusion}

The increasing awareness of TMA pathogenesis and its risk factors - despite rare - is of growing interest among the transplant community. The proper preparation of the KTR, particularly as regard genetic mutations abnormalities related to the evolution of aHUS (complement-mediated HUS) is a fundamental step before commencing the transplant. This is particularly crucial in ESRF patients due to HUS disease. On the other hand, the perfect evaluation of KTR can result in detection of a simple avoidable etiology for HUS e.g., medications, switching of which can result in a complete disease reversal and avoidance of unnecessary costly medications. The latter, however, is under robust investigations to reduce their cost and improve the cost-effectiveness balance e.g., "therapy upon recurrence" strategy. However, more efforts still warranted to simplify these complicated therapeutic strategies of this devastating disease and guarantee better patient and graft outcome.

\section{Acknowledgments}

None.

\section{Conflicts of interest}

The author declares there is no conflict of interest.

\section{References}

1. Shochet 1, Kanellis j, Simpson I, et al. De novo thrombotic microangiopathy following simultaneous pancreas and kidney transplantation managed with eculizumab. Nephrology. 2017;22(Suppl 1):23-27.

2. Shulman H, Striker G, Deeg HJ, et al. Nephrotoxicity of cyclosporine A after allogeneic marrow transplantation: Glomerular thromboses and tubular injury. N Engl J Med. 1981;305(23)::1392-1395.

3. Schwimmer J, Nadasdy TA, Spitalnik PF, et al. De novo thrombotic microangiopathy in renal transplant recipients: A comparison of hemolytic uremic syndrome with localized renal thrombotic microangiopathy. Am J Kidney Dis. 2003;41(2):471-479.

4. Aiyangar A, Kiran K, Prasad R, et al. Thrombotic microangiopathy in renal allografts. Indian Journal of Nephrology. 2014;24(1):24-32.

5. Noris M, Caprioli J, Bresin E, et al. Relative role of genetic complement abnormalities in sporadic and familial aHUS and their impact on clinical phenotype. Clin J Am Soc Nephrol. 2010;5:1844-1859.

6. Noris M, Remuzzi G. Thrombotic microangiopathy after kidney transplantation. Am J Transplant. 2010;10:1517-1523.

7. Cofiell R, Kukreja A, Bedard K, et al. Eculizumab reduces complement activation, inflammation, endothelial damage, thrombosis, and renal injury markers in aHUS. Blood. 2015;125(21):3253-3262.
8. Dedhia P, Govil A, Mogilishetty G, et al. Eculizumab and Belatacept for De Novo Atypical Hemolytic Uremic Syndrome Associated With CFHR3-CFHR1 Deletion in a Kidney Transplant Recipient: A Case Report. Transplantation Proceedings. 2017;49(1):188-192.

9. Noris M, Remuzzi G. Atypical hemolytic-uremic syndrome. $N$ Engl $J$ Med. 2009;361(17):1676-1687.

10. Zarifian A, Meleg-Smith S, O'donovan R, et al. Cyclosporineassociated thrombotic microangiopathy in renal allografts. Kidney Int. 1999;55:2457-2466.

11. Ruggenenti P. Post-transplant hemolytic-uremic syndrome. Kidney Int. 2002;62(3):1093-1104.

12. Kwon O, Hong SM, Sutton TA, et al. Preservation of peritubular capillary endothelial integrity and increasing pericytes may be critical to recovery from post-ischemic acute kidney injury. Am J Physiol Renal Physiol. 2008;295:F351-F359.

13. Murer L, Zacchello G, Bianchi D, et al. Thrombotic microangiopathy associated with parvovirus B 19 infection after renal transplantation. $J \mathrm{Am}$ Soc Nephrol. 2000;11(6):1132-1137.

14. Waiser J, Budde K, Rudolph B, et al. De novo hemolytic uremic syndrome postrenal transplant after cytomegalovirus infection. Am J Kidney Dis. 1999;34(3):556-559.

15. Olie KH, Goodship TH, Verlaak R, et al. Posttransplantation cytomegalovirus-induced recurrence of atypical hemolytic uremic syndrome associated with a factor $\mathrm{H}$ mutation: successful treatment with intensive plasma exchanges and ganciclovir. Am J Kidney Dis. 2005;45:e12-e15.

16. Ardalan MR, Shoja MM, Tubbs RS, et al. Postrenal transplant hemophagocytic lymphohistiocytosis and thrombotic microangiopathy associated with parvovirus b19 infection. Am $J$ Transplant. 2008;8(6):1340-1344

17. Le Quintrec M, Lionet A, Kamar N, et al. Complement mutationassociated de novo thrombotic microangiopathy following kidney transplantation. Am J Transplant. 2008;8(8):1694-1701.

18. Miller RB, Burke BA, Schmidt WJ, et al. Recurrence of haemolyticuraemic syndrome in renal transplants: a single-centre report. Nephrol Dial Transplant. 1997;12(7):1425-1430.

19. Miller BW, Hmiel SP, Schnitzler MA, et al. Cyclosporine as cause of thrombotic microangiopathy after renal transplantation. Am J Kidney Dis. 1997;29(5):813-814.

20. Karthikeyan V, Parasuraman R, Shah V, et al. Outcome of plasma exchange therapy in thrombotic microangiopathy after renal transplantation. $\mathrm{Am} \mathrm{J}$ Transplant. 2003;3(10):1289-1394.

21. Holman MJ, Gonwa TA, Cooper B, et al. FK506-associated thrombotic thrombocytopenic purpura. Transplantation. 1993;55(1):205-206.

22. Trimarchi HM, Truong LD, Brennan S, et al. FK506-associated thrombotic microangiopathy: report of two cases and review of the literature. Transplantation. 1999;67(4):539-544.

23. Pham PT, Peng A, Wilkinson AH, et al. Cyclosporine and tacrolimusassociated thrombotic microangiopathy. Am $J$ Kidney Dis. 2000;36(4):844-850.

24. Zuber J, Le Quintrec M, Sberro-Soussan R, et al. New insights into postrenal transplant hemolytic uremic syndrome. Nat Rev Nephrol. 2011;7(1):23-35.

25. Agarwal A, Mauer SM, Matas AJ, et al. Recurrent hemolytic uremic syndrome in an adult renal allograft recipient: current concepts and management. J Am Soc Nephrol. 1995;6(4):1160-1169. 
26. Hebert D, Sibley RK, Mauer SM. Recurrence of hemolytic uremic syndrome in renal transplant recipients. Kidney Int Suppl. 1986;19:S51S58.

27. Artz MA, Steenbergen EJ, Hoitsma AJ, et al. Renal transplantation in patients with hemolytic uremic syndrome: high rate of recurrence and increased incidence of acute rejections. Transplantation. 2003;76(5):821826.

28. Oyen O, Strøm EH, Midtvedt $\mathrm{K}$, et al. Calcineurin inhibitor-free immunosuppression in renal allograft recipients with thrombotic microangiopathy/hemolytic uremic syndrome. Am J Transplant. 2006;6(2):412-418.

29. https://www.uptodate.com/recurrent-and-de-novo-hus-after-renaltransplantation.

30. Ducloux D, Rebibou JM, Semhoun-Ducloux S, et al. Recurrence of hemolytic-uremic syndrome in renal transplant recipients: a metaanalysis. Transplantation. 1998;65(10):1405-1407.

31. Bresin E, Daina E, Noris M, et al. Outcome of renal transplantation in patients with non-Shiga toxin-associated hemolytic uremic syndrome: prognostic significance of genetic background. Clin J Am Soc Nephrol. 2006;1(1):88-99.

32. Lahlou A, Lang P, Charpentier B, et al. Hemolytic uremic syndrome. Recurrence after renal transplantation. Groupe Coopératif de l'Ile-deFrance (GCIF). Medicine (Baltimore). 2000;79(2):90-102.

33. Karpman D, Sartz L, Johnson S. Pathophysiology of typical hemolytic uremic syndrome. Semin Thromb Hemost. 2010;36(6):575-585.

34. Satoskar AA, Pelletier R, Adams P, et al. De novo thrombotic microangiopathy in renal allograft biopsies-role of antibody-mediated rejection. American Journal of Transplantation. 2010;10(8):1804-1811.

35. Chua JS, Baelde HJ, Zandbergen M, et al. Complement Factor C4d Is a Common Denominator in Thrombotic Microangiopathy. J Am Soc Nephrol. 2015;26(9):2239-2247.

36. Wrenn SM, Gibson PC, Hain DS, et al. Development of de novo Hemolytic Uremic Syndrome Post-Transplant and the Role of Donor Specific Antibodies: A Case Report and Review of Literature. J immuno Biol. 2015;1:101.

37. Broeders E, Stordeur P, Rorive S, et al. A "silent", new polymorphism of factor $\mathrm{H}$ and apparent de novo atypical haemolytic uraemic syndrome after kidney transplantation. Case Reports. 2014;bcr2014207630.

38. Kaplan BS, Ruebner RL, Spinale JM, et al. Current treatment of atypical hemolytic uremic syndrome. Intractable Rare Dis Res. 2014;3(2):34-45.

39. Delvaeye M, Noris M, De Vriese A, et al. Mutations in thrombomodulin in hemolytic-uremic syndrome. N Engl J Med. 2009;361(4):345-357.

40. Reynolds JC, Agodoa LY, Yuan CM, et al. Thrombotic microangiopathy after renal transplantation in the United States. Am J Kidney Dis. 2003;42(5):1058-1068.

41. Rolla D, Fontana I, Ravetti JL, et al. De Novo Post-transplant Thrombotic Microangiopathy Localized Only to the Graft in APKD with Thrombophilia. J Kidney. 2015;1:101.

42. Loirat C, Fremeaux-Bacchi V. Hemolytic uremic syndrome recurrence after renal transplantation. Pediatr Transplant. 2008;12(6):619-629.

43. Caprioli J, Noris M, Brioschi S, et al. Genetics of HUS: The impact of MCP, CFH, and IF mutations on clinical presentation, response to treatment, and outcome. Blood. 2006;108(4):1267-1279.

44. Sellier-Leclerc AL, Fremeaux-Bacchi V, Dragon-Durey MA, et al. Differential impact of complement mutations on clinical characteristics in atypical hemolytic uremic syndrome. J Am Soc Nephrol. 2007;18(8):23922400
45. Seitz B, Albano L, Vocila F et al. Recurrence of hemolytic uremic syndrome after renal transplantation. Transplant Proc. 2007;39(8):25832585

46. Bren A, Pajek J, Grego K, et al. Follow-up of kidney graft recipients with cyclosporine-associated hemolytic-uremic syndrome and thrombotic microangiopathy. Transplant Proc. 2005;37(4):1889-1891.

47. Donne RL, Abbs I, Barany P, et al. Recurrence of hemolytic uremic syndrome after live related renal transplantation associated with subsequent de novo disease in the donor. Am J Kidney Dis. 2002;40(6):E22.

48. Kwon T, Dragon-Durey MA, Macher MA, et al. Successful pretransplant management of a patient with anti-factor $\mathrm{H}$ autoantibodiesassociated haemolytic uraemic syndrome. Nephrol Dial Transplant. 2008;23(6):2088-2090.

49. Le Quintrec M, Zuber J, Noel LH, et al. Anti-Factor H autoantibodies in a fifth renal transplant recipient with atypical hemolytic and uremic syndrome. Am J Transplant. 2009;9(5):1223-1229.

50. Kaplan BS, Meyers KE, Schulman SL. The pathogenesis and treatment of hemolytic uremic syndrome. J Am Soc Nephrol. 1998;9(6):1126-1133.

51. Le Quintrec M, Zuber J, Moulin B et al. Complement genes strongly predict recurrence and graft outcome in adult renal transplant recipients with atypical hemolytic and uremic syndrome. Am J Transplant. 2013;13(3):663- 675

52. Fremeaux-Bacchi V, Fakhouri F, Garnier A, et al. Genetics and outcome of atypical hemolytic uremic syndrome: a nationwide French series comparing children and adults. Clin J Am Soc Nephrol. 2013;8(4):554 562.

53. Legendre CM, Licht $\mathrm{C}$, Muus $\mathrm{P}$ et al. Terminal complement inhibitor eculizumab in atypical hemolytic-uremic syndrome. $N \mathrm{Engl} \mathrm{J} \mathrm{Med}$. 2013;368:2169-2181.

54. Zuber J, Le Quintrec M, Krid S et al. Eculizumab for atypical hemolytic uremic syndrome recurrence in renal transplantation. Am J Transplant. 2012;12(12):3337-3354.

55. Ardissino G, Possenti I, Tel F et al. Discontinuation of eculizumab treatment in atypical hemolytic uremic syndrome: an update. Am J Kidney Dis. 2015;66(1):172-173.

56. National Institute for Health and Care Excellence. Eculizumab for treating atypical haemolytic uraemic syndrome. London, 2015. p. 1-46.

57. Ashman N, Chapagain A, Dobbie H, et al. Belatacept as maintenance immunosuppression for postrenal transplant de novo drug-induced thrombotic microangiopathy. Am J Transplant. 2009;9(2):424-427.

58. Midtvedt K, Bitter J, Dørje C, et al. Belatacept as immunosuppression in patient with recurrence of hemolytic uremic syndrome after renal transplantation. Transplantation. 2009;87:901.

59. Watt T, Warshaw B, Katzenstein HM. Atypical hemolytic uremic syndrome responsive to steroids and intravenous immune globulin. Pediatr Blood Cancer. 2009;53(1):90-91.

60. Lionet A, Provôt F, Glowacki F, et al. A case of adult atypical haemolytic uraemic syndrome related to anti-factor $\mathrm{H}$ autoantibodies successfully treated by plasma exchange, corticosteroids and rituximab. NDT Plus. 2009;2(6):458-460.

61. Larrea CF, Cofan F, Oppenheimer F, et al. Efficacy of eculizumab in the treatment of recurrent atypical hemolytic-uremic syndrome after renal transplantation. Transplantation. 2010;89(7):903-904.

62. Nürnberger J, Philipp T, Witzke $O$, et al. Eculizumab for atypical hemolytic-uremic syndrome. N Engl J Med. 2009;360:542-544.

63. Châtelet V, Lobbedez T, Frémeaux-Bacchi V, et al. Eculizumab: safety and efficacy after 17 months of treatment in a renal transplant patient with 
recurrent atypical hemolytic-uremic syndrome: case report. Transplant Proc. 2010;42(10):4353-4355.

64. Davin JC, Gracchi V, Bouts A, et al. Maintenance of kidney function following treatment with eculizumab and discontinuation of plasma exchange after a third kidney transplant for atypical hemolytic uremic syndrome associated with a CFH mutation. Am J Kidney Dis. 2010;55(4):708-711.
65. Wong E, Challis R, Sheerin N, et al. Patient stratification and therapy in atypical haemolytic uraemic syndrome (aHUS). Immunobiology. 2016;221:715-718.

66. Brand JA, Verhave JC, Adang EM, et al. Cost-effectiveness of eculizumab treatment after kidney transplantation in patients with atypical haemolytic uraemic syndrome. Nephrol Dial Transplant. 2017;32:i115-i122. 